\title{
Evaluation of Upper Endoscopic Findings before and after Transjugular Intrahepatic Portosystemic Shunt in Egyptian Patients with Budd-Chiari Syndrome
}

\author{
Ahmed S. Abdelmoaty ${ }^{1}$, Mohammad A. Sakr ${ }^{1}$, Mohamed E. Aboelmaaty ${ }^{2}$, \\ Hend E. Ebada ${ }^{1}$ \\ ${ }^{1}$ Department of Tropical Medicine, Faculty of Medicine, Ain Shams University, Cairo \\ 11341, Egypt \\ ${ }^{2}$ Department of Radiodiagnosis and Interventional Radiology, Faculty of Medicine, \\ Ain Shams University, Cairo 11341, Egypt
}

Corresponding Author Ahmed S. Abdelmoaty

\section{Mobile:}

01002873845

\section{E mail:}

ahmedsamir@med.asu. edu.eg

Key words:

Portal hypertension, Gastroesophageal varices, Transjugular Intrahepatic

Portosystemic Shunt, Budd-Chiari syndrome, portal hypertensive gastropathy
Background and study aim: Successful transjugular intrahepatic portosystemic shunt (TIPS) insertion effectively lowers the portosystemic pressure gradient, resolves ascites and improves esophageal varices, we studied the effect of TIPS on the grades of gastroesophageal varices and portal hypertensive gastropathy (PHG) in Egyptian patients with BuddChiari Syndrome (BCS).

Patients and Methods: This prospective study was conducted at the Tropical Medicine Department; Ain Shams University on 20 patients with confirmed BCS with esophageal, gastric varices and PHG. Upper GI endoscopy was conducted before TIPS. Patients were followed up by upper GIT endoscopy at one and six months after TIPS.
Results: After TIPS, there was a highly significant improvement of portal pressure, (before TIPS: Mean \pm SD $=24.9 \pm 1.9$; after TIPS: $8.3 \pm 1.5$, p-value $<0.001)$ and esophageal varices $(\mathrm{EV})$. In two of three patients with large EV within one-month post-TIPS and in all 3 patients within 6 months post TIPS. 15 patients with medium-sized EV markedly improved to 7 patients reduced to small $\mathrm{EV}$ and one reduced to non $\mathrm{EV}$ in one month and 9 patients to small EV and 6 reduced to non EV after 6 months and all 2 patients with small EV reduced to non EV after one and six months. After TIPS, there was a highly significant improvement of PHG in most cases.

Conclusion: Successful TIPS insertion in BCS patients leads to improvement in esophageal varices \& PHG.

According to the duration of symptoms and signs of liver disease, BCS can be presented with acute, subacute or chronic form; the most common presentation is the chronic form. A high index of suspicion is necessary for diagnosis because clinical manifestations and laboratory results are nonspecific [3].

Radiological imaging is enough to confirm a diagnosis of BCS. A liver biopsy is needed only if imaging not conclusive with high suspicious of BCS. Doppler ultrasonography, computed tomography (CT), magnetic resonance imaging (MRI) and hepatic venography are the most useful teachings [4]. 
Medical treatment of BCS is aimed at prevention of extension and recurrence of thrombosis. This includes anticoagulation therapy along with treatment of underlying cause of thrombophilia. Around ten percent of BCS patients can be managed medically (anticoagulation with or without thrombolytic therapy, and diuretics). However, most of patients managed with more invasive procedures to improve hepatic outflow obstruction, including percutaneous hepatic vein dilatation with or without stenting, (TIPS) or shunt surgery [5].

Upper GI endoscopy is recommended in every case of BCS; not only for diagnosis of complications of portal hypertension like EV or PHG; but also, for the eradication of any risky varices as patients with BCS should start anticoagulation therapy once diagnosis is made. Anticoagulation therapy in the presence of risky forces would carry greater risk of bleeding. Dabbous and collagues, found that large-sized varices occurred in $40 \%$ of Egyptian patients with BCS at time of diagnosis, $40 \%$ for mediumsized and 20\% for small-sized varices [6].

Successful TIPS insertion effectively lowers the portosystemic pressure gradient, resolves ascites and improves EV, PHG, liver and renal function [7]. The repeated follow-up endoscopy implies a considerable burden on endoscopies and related costs as they require that patients repeatedly undergo an unpleasant invasive procedure especially if fully anticoagulated. On the other hand, many patients refuse repeated endoscopies because of discomfort and fear of transmission of or contribute to infection as it is associated with disruption of the natural barriers [8]. Therefore, a considerable interest in decreasing the frequency of follow-up upper GI endoscopy after TIPS by evaluation of its effect on esophagogastric varices and PHG may be needed.

The aim of this study was to evaluate the effect of TIPS -as a decompression technic on the portal hypertension as reflected by grades of esophageal \& gastric varices and grades of portal hypertensive gastropathy (PHG) in Egyptian patients with BCS.

\section{PATIENTS AND METHODS}

Type of the study: prospective cohort study

Populations: twenty patients with primary chronic BCS who have esophageaogastric varices and PHG on upper endoscopy during the period from September 2018 to September 2019 and eligible criteria for radiological intervention (patients with primary BCS who were not responding to medical treatment \pm angioplasty/ stenting of IVC or hepatic veins for short length stenosis or upstream dilatation or having all hepatic veins occluded from the start). They were managed by Budd-Chiari Study Group in Tropical Medicine Department, Ain Shams University Hospitals.

All participants were subjected to: Detailed history taking, Physical examination with special stress on symptoms and signs of chronic liver disease and portal hypertension and imaging evaluation by abdominal Duplex/US using Mindray M7 ultrasound machine with special stress on the patency of hepatic vasculature (hepatic veins (HVs), portal vein and inferior vena cava)

All patients underwent TIPS with uncovered stents.

Exclusion Criteria: secondary BCS e.g.: tumors; IVC obstruction; portal vein thrombosis; associated liver disease (e.g.: HCV, HBV); primary hepatic tumors; congestive heart failure and severe pulmonary hypertension); severe bleeding tendency $(\mathrm{INR}>5)$ and thrombocytopenia (platelets <20.000); biliary obstruction; uncontrolled infection.

All patients provided written informed consent. Assessment by upper endoscopy two weeks before the intervention to asses portal hypertension related changes (i.e. Status of esophageal and gastric varices and PHG).

Oral anticoagulation therapy was stopped five days before the procedure with bridging therapy (LMWH); to be stopped before TIPS 12-24 h. Prophylactic antibiotic were administered for all patients one hour before TIPS.

\section{Technical considerations}

TIPS creation was carried out using "Direct Intrahepatic Portocaval Shunt (DIPS) technique." All procedures were done under general anesthesia. The right internal jugular vein was punctured under sonographic guidance followed by insertion of a long 10 French sheath of TIPS set (Cook, USA) over a hydrophilic guidewire 0.035 " (Terumo, Japan) down to the retrohepatic portion of IVC. Using real-time US guidance an $18 \mathrm{G} 20 \mathrm{~cm}$ needle (HS company, Italy) was

Abdelmoaty et al., Afro-Egypt J Infect Endem Dis 2021;11 (1):18-26

https://aeji.journals.ekb.eg/

http://mis.zu.edu.eg/ajied/home.aspx 
introduced after percutaneous puncture through intercostal approach into liver parenchyma and advanced till puncturing the right portal branch in an intrahepatic position 1-2 cm distal to portal bifurcation then the needle is advanced through the liver parenchyma till puncturing the retrohepatic IVC, to create a track between the right portal branch and IVC. A Amplatz super stiff guidewire 0.035" (Boston, USA) was then introduced through the puncture needle so that the tip of the guidewire reached the IVC then snared using a dormia basket (Cook, USA) through the long sheath inserted through the transjugular route and withdrawn till it came out of the sheath at the neck. The puncture needle was withdrawn beyond the right portal branch, and the track between the IVC and right portal branch was dilated over the wire by $8 \mathrm{~mm}$ balloon (Boston, USA). The sheath was then advanced down till it reached the right portal branch. A 5 French catheter (Cobra 2 catheter) (Wanda, Boston, USA) was introduced beside the Super stiff guidewire through the sheath over a hydrophilic guidewire. The catheter was advanced down and manipulated to reach the main PV, and portography was obtained. The stiff guidewire was then withdrawn out of the sheath and introduced through the Cobra catheter to reach the main PV. The catheter was withdrawn, and then a $10 \mathrm{~mm}$ diameter self-expandable bare metallic stent (Wallstent, Boston Scientific, USA) was introduced over the wire. The long sheath was withdrawn upward till it reached the IVC so that the stent can be deployed and adjusted with lower end at the main PV just below its bifurcation and upper end at the retrohepatic IVC. The stent was dilated using $10 \mathrm{~mm}$ balloon (Wanda, Boston, USA) to get its maximum diameter, and then contrast was injected through the stent to ensure its patency. The puncture track was embolized through the puncture needle by coil (Cook, USA) to prevent from intraperitoneal bleeding. The pressure gradient was measured between the PV and IVC before and after stent insertion to ensure satisfactory reduction of pressure gradient to $<10$ $\mathrm{mmHg}$

Patients were hospitalized for one week after the TIPS for detection and management of any complications (sepsis, hepatic encephalopathy, bleeding and stent dysfunction) and adjustment of anticoagulation therapy. Antibiotic prophylaxis was administered for all patients (1$2 \mathrm{~h})$ before intervention in the form of a combination of ampicillin-sulbactam $1.5 \mathrm{~g}$ intravenous (IV) and cefotaxime $1 \mathrm{~g}$ IV. Oral warfarin therapy has been introduced together with parenteral anticoagulation (LMWH after 24 hours) till INR reaches (2-3) then oral therapy to be continued lifelong. Duplex U/S has been performed to detect stent patency at days $(1,3$, and 7).

Upper gastrointestinal endoscopy were subjected to all Patients two weeks before and one month after TIPS to assess signs of portal hypertension in the form of grading of esophageal varices according to the American Association for the Study of Liver Diseases (AASLD) practice guideline [9].

PHG is classified according to Baveno IIIscoring system (B) [10]

\section{Statistical Analysis}

The qualitative data were presented as number and percentages while the quantitative data was presented as mean, standard deviations (SD), and ranges. Analysis of variance (ANOVA) was used to test the difference between mean values. Multiple comparisons between pairs of groups were performed using LSD (Post hoc range test) where results were presented as mean and SD. Chi-Square test $\chi^{2}$ and Fisher's Exact Test were used to test the difference in proportions of variables among the presentations. All data were analyzed using SPSS version 17. A P-value less than 0.05 was considered significant (S); a Pvalue less than 0.01 was considered highly significant (HS), and a P-value less than 0.001 was considered very highly significant (VHS).

\section{RESULTS}

Our results showed that BCS more predominant in female patients than male patients $(55 \%$ versus $45 \%$ respectively). All of the patients in the current study were diagnosed during the chronic phase of the disease (100\%). According to Child-Pugh score, $30 \%$ of patients were Child A, $70 \%$ of patients were Child B and none of them was Child C. Regarding the etiology, we identified combined etiology (multifactorial) in $25 \%$ of the patients. Factor V Leiden mutation (FVLM) was the most common cause and this was documented in $50 \%$ of patients. The second most common cause in our series was antiphospholipid antibody syndrome (APLAS) and this was found in $30 \%$ of patients. Hormonal

\footnotetext{
Abdelmoaty et al., Afro-Egypt J Infect Endem Dis 2021;11(1):18-26

https://aeji.journals.ekb.eg/

http://mis.zu.edu.eg/ajied/home.aspx
} 
therapy was encountered in $63.5 \%$ of female patients. The relevant symptoms in the 20 patients with BCS at the time of presentation were abdominal enlargement, abdominal pain, lower limb swelling, upper GIT bleeding and jaundice in $95 \%, 70 \%, 30 \%, 15 \%$ and $10 \%$ of patients respectively. Regarding the signs, hepatomegaly, tender liver, ascites, splenomegaly, dilated veins over the trunk and body, jaundice and hepatic encephalopathy in $85 \%, 60 \%, 95 \%, 60 \%, 25 \%, 10 \%$, and $10 \%$ of patients respectively. Most of the cases had normal serum albumin \{mean serum albumin was $3.54 \pm 0.54 \mathrm{~g} / \mathrm{L}$ (range $3-4 \mathrm{~g} / \mathrm{L}$ ) \}. Table (1) shows baseline radiological criteria of the studied patients. Caudate lobe hypertrophy was detected in $(75 \%)$, mottling appearance of the liver in $(85 \%)$, regenerative nodules in $(20 \%)$ and intrahepatic collaterals were detected in $(80 \%)$ of patients. Screening upper GI endoscopy was done for all patients 2 weeks before TIPS to evaluate the status of varices and PHG. EV were found in all patients (100\%), where large EV were found in $(15 \%)$ of pateints, $(75 \%)$ had medium-sized EV and (10\%) had small- sized EV. Fundal varices were detected in $(5 \%)$. All recruited patients $(100 \%)$ had PHG, where $(65 \%)$ had severe PHG and (35\%) had mild PHG. In the present study, we have recruited 20 patients, who have occlusion of all hepatic veins that enabled us to perform TIPS in all cases. The mean portal pressure showed highly significant reduction after stent insertion denoting successful TIPS insertion table 5. The Patency rate of TIPS was $90 \%$ at 6 months. Patients after TIPS who needed stent revisions by balloon angioplasty were two (10\%); one patient at 3 months and the other at 6 months of follow-up. Post-TIPS transient right upper quadrant abdominal pain and tenderness (lasting 2-3 days after the procedure) was encountered in all studied patients. There was a dramatic improvement of abdominal enlargement, ascites, abdominal pain, hepatic tenderness and abdominal dilated veins after TIPS which improved soon in the first few days after the intervention (table3). All patients had normal kidney function before intervention to be fit for contrast during TIPS insertion and all patients had normal renal profile after intervention. A significant decrease in ALT and serum bilirubin levels as well as a significant increase in serum albumin levels after TIPS (table 3), with subsequent improvement of the Child score after TIPS (Table 4). The mean size of the liver and spleen $(\mathrm{cm})$ at the end of followup was significantly decreased when compared with that before the intervention. The mean portal vein diameter was significantly decreased and the mean portal vein velocity was significantly increased at the end of followup. The frequency of hepato-petal portal vein flow direction was significantly increased at the end of follow-up (Table 5). After TIPS, there was a highly statistically significant improvement of EV. In two of three patients with large EV within one-month post- TIPS (2 reduced to medium sized) and in all 3 patients within 6 months post-TIPS ( 2 large reduced to medium sized and one to small EV). 15 patients with medium sized EV markedly improved in 7 patients reduced to small EV and one reduced to non EV in one month and 9 patients with small $\mathrm{EV}$ and 6 reduced to non EV after 6 months and all 2 patients with small EV reduced to non EV after one and six months. Isolated gastric varix persisted at one and six months. Also, after TIPS, there was a highly statistically significant improvement of PHG in most cases. In 9 of 13 patients with severe PHG within one-month postTIPS (reduced to mild disease) and in 12 of 13 within 6 months post-TIPS ( 6 reduced to mild disease and 6 reduced to none). All 7 patients with mild PHG markedly improved to non PHG at one and six months (table 6). 
Table (1): Baseline radiological criteria of the studied patients; using duplex Ultrasonography.

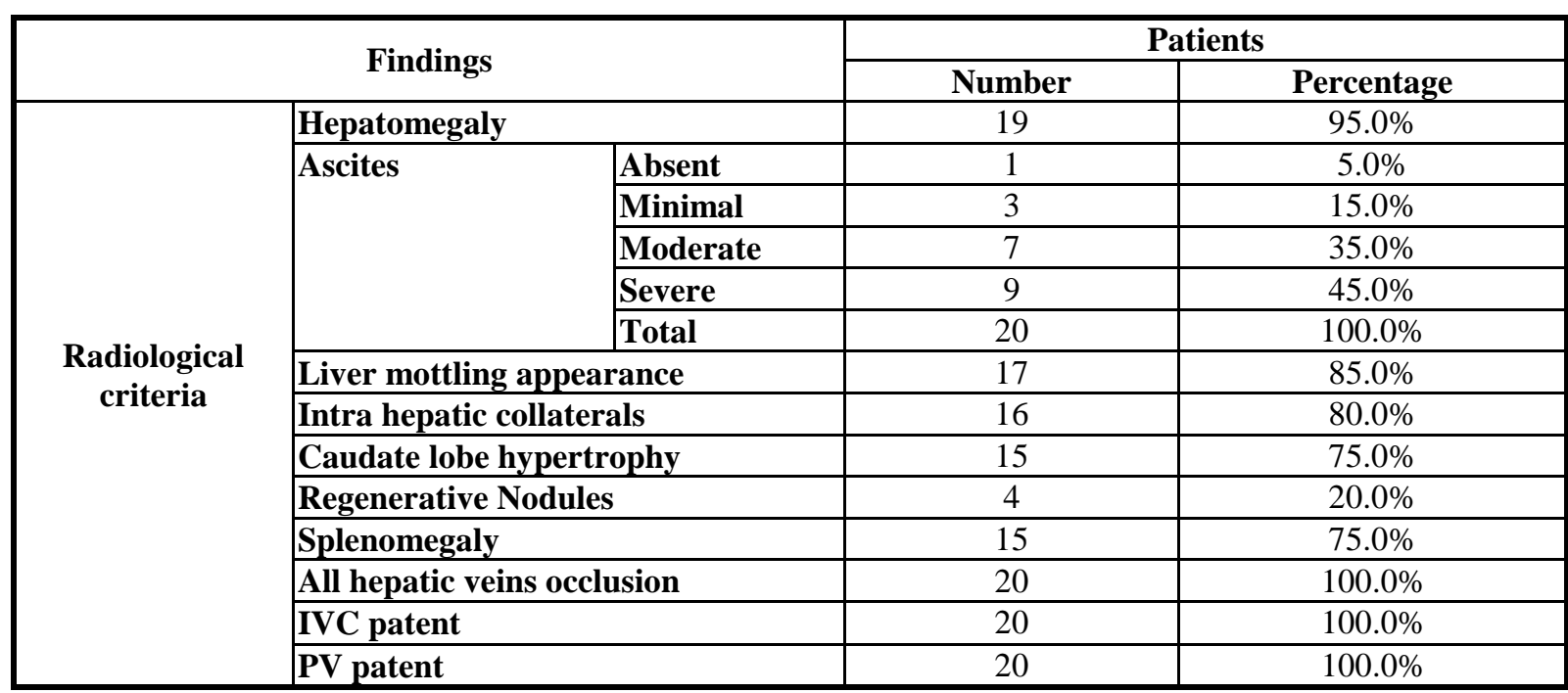

IVC Inferior vena cava

PV portal vein

Table (2): Comparison between clinical findings before and after intervention.

\begin{tabular}{|c|c|c|c|c|c|c|c|c|c|c|c|}
\hline & \multicolumn{2}{|c|}{ Before } & \multicolumn{3}{|c|}{1 month } & \multicolumn{3}{|c|}{3 months } & \multicolumn{3}{c|}{6 months } \\
\hline & NO & $\boldsymbol{\%}$ & NO & $\%$ & P value & NO & \% & P value & NO & \% & P value \\
\hline Abdominal pain & 14 & 70 & 9 & 45 & 0.062 & 7 & 35 & $0.016^{*}$ & 2 & 10 & $0.000^{*}$ \\
\hline Abdominal Tenderness & 16 & 80 & 2 & 10 & $0.000^{*}$ & 1 & 5 & $0.000^{*}$ & 1 & 5 & $0.000^{*}$ \\
\hline Abdominal Enlargement & 19 & 95 & 11 & 55 & $0.008^{*}$ & 7 & 35 & $0.000^{*}$ & 2 & 10 & $0.000^{*}$ \\
\hline Ascites & 19 & 95 & 9 & 45 & $0.002^{*}$ & 7 & 35 & $0.000^{*}$ & 4 & 20 & $0.000^{*}$ \\
\hline Dilated veins on the abdomen & 5 & 25 & 2 & 10 & 0.250 & 1 & 5 & 0.125 & 1 & 5 & 0.055 \\
\hline
\end{tabular}

${ }^{\wedge} \mathrm{McNemar}$ test (In relation to before),

Table (3): Laboratory investigations before intervention, during and at the end of follow up (at 1, 3, 6 months).

\begin{tabular}{|l|c|c|c|c|c|c|}
\hline \multicolumn{1}{|c|}{ Time } & $\begin{array}{c}\text { Serum } \\
\text { Albumin } \\
(\mathbf{g} / \mathbf{d L}) \\
\text { Mean+SD }\end{array}$ & $\begin{array}{c}\text { AST (mg/dL) } \\
\text { Median (IQR) }\end{array}$ & $\begin{array}{c}\text { ALT (mg/dL) } \\
\text { Median (IQR) }\end{array}$ & $\begin{array}{c}\text { Bilirubin (mg/dL) } \\
\text { Median } \\
(\text { IQR) }\end{array}$ & $\begin{array}{c}\text { PTT } \\
\text { (seconds) } \\
\text { Mean } \pm \text { SD }\end{array}$ & $\begin{array}{c}\text { PT } \\
\text { (seconds) } \\
\text { Mean } \pm \text { SD }\end{array}$ \\
\hline Before & $3.49 \pm 0.499$ & $32(28)$ & $44(14)$ & $1.1(0.8)$ & $45.21 \pm 11.59$ & $13.53 \pm 2.09 \bullet$ \\
\hline $\mathbf{1 ~} \mathbf{~ m}$ & $4.07 \pm 0.391$ & $23(12)$ & $23(17)$ & $1(0)$ & $44.63 \pm 11.39$ & $22.05 \pm 6.65 \bullet$ \\
\hline $\mathbf{3 ~} \mathbf{~ m}$ & $4.12 \pm 0.302$ & $38(26)$ & $23(11)$ & $0.7(0)$ & $42.53 \pm 10.17$ & $24.24 \pm 5.79 \bullet$ \\
\hline $\mathbf{6 ~} \mathbf{~ m}$ & $4.05 \pm 0.378$ & $35(25)$ & $21(17)$ & $0.86(0)$ & $46.74 \pm 10.96$ & $23.15 \pm 5.31 \bullet$ \\
\hline $\mathbf{p}$-value & $0.000^{*}$ & 0.968 & $0.000^{*}$ & $0.002^{*}$ & 0.761 & $0.000^{*}$ \\
\hline
\end{tabular}

- Patients are kept on warfarin $\mathrm{m}=$ month

*p-value: comparison between before and after 1 year ( $\mathrm{P}$ value was significant at the level of 0.01 ).

Table (4): Comparison between Child class before and 6 months after intervention in the studied patients .

\begin{tabular}{|l|c|c|c|c|c|c|}
\hline \multirow{2}{*}{ Child Class } & \multicolumn{2}{|c|}{ Before } & \multicolumn{2}{c|}{ After } & \multirow{2}{*}{$\mathbf{X}^{\mathbf{2}}$} & \multirow{2}{*}{ P-value } \\
\cline { 2 - 5 } & No. & $\mathbf{\%}$ & No. & $\mathbf{\%}$ & & \multirow{2}{*}{12.379} \\
\cline { 1 - 5 } Class A & 6 & $30 \%$ & 17 & $85 \%$ & \multirow{2}{*}{0.002} \\
\hline Class B & 14 & $70 \%$ & 3 & $15 \%$ & & \\
\hline Class C & 0 & $0 \%$ & 0 & $0 \%$ & & \\
\hline
\end{tabular}

Abdelmoaty et al., Afro-Egypt J Infect Endem Dis 2021;11(1):18-26

https://aeji.journals.ekb.eg/

http://mis.zu.edu.eg/ajied/home.aspx 


\begin{tabular}{|l|l|l|l|l|l|l|}
\hline Total & 20 & $100 \%$ & 20 & $100 \%$ & & \\
\hline
\end{tabular}

Table (5): Comparison between radiological findings before and after intervention .

\begin{tabular}{|c|c|c|c|c|c|c|c|}
\hline & \multicolumn{2}{|c|}{ Before TIPS } & \multicolumn{2}{|c|}{6 Months after TIPS } & \multirow[t]{2}{*}{$\mathbf{t}$} & \multirow[t]{2}{*}{ P-value } \\
\hline & & Mean \pm SD & Rang & Mean \pm SD & Rang & & \\
\hline \multicolumn{2}{|l|}{ size of the liver $(\mathrm{cm})$} & $18.85 \pm 2.01$ & $14-22$ & $17.50 \quad 1.43$ & $15-20$ & 5.107 & 0.000 \\
\hline \multicolumn{2}{|l|}{ size of the spleen $(\mathrm{cm})$} & $14.65 \pm 3.38$ & $9-21$ & 13.65 & $9-18$ & 5.210 & 0.000 \\
\hline \multicolumn{2}{|l|}{ Portal vein diameter (mm) } & $10.82 \pm 2.52$ & $6-16$ & $9.0 \pm 1.6$ & $6-12$ & 5.102 & $0.009^{*}$ \\
\hline \multicolumn{2}{|l|}{ Portal vein velocity $(\mathrm{cm} / \mathrm{sec})$} & $14.55 \pm 4.87$ & $7-25$ & $31.75 \pm 8.79$ & $17-45$ & -9.978 & 0.000 \\
\hline \multirow{3}{*}{ Portal vein flow direction } & & No. & $\%$ & No. & $\%$ & & \multirow{3}{*}{0.001} \\
\hline & Petal & 6 & $30.0 \%$ & 18 & $90.0 \%$ & & \\
\hline & $\overline{\text { Fugal }}$ & 14 & $70.0 \%$ & 2 & $10.0 \%$ & & \\
\hline *Portal vein pressure $(\mathrm{mm} / \mathbf{H g})$ & & $24.9 \pm 1.9$ & & & $8.3 \pm 1.5$ & & 0.001 \\
\hline
\end{tabular}

*The mean portal pressure showed highly significant reduction after stent insertion denoting successful TIPS insertion (before TIPS: Mean $\pm \mathrm{SD}=24.9 \pm 1.9$; immediate after TIPS: $8.3 \pm 1.5$, p value $<0.001$ ) ).

Table (6): Comparison between upper endoscopic findings before and after intervention in the studied patients.

\begin{tabular}{|c|c|c|c|c|c|c|c|c|c|}
\hline \multicolumn{4}{|c|}{$\begin{array}{ll}\text { Findings } \\
\end{array}$} & \multicolumn{2}{|c|}{ Before } & \multirow{2}{*}{\begin{tabular}{|l|} 
After 1m \\
$3(15 \%)$ \\
\end{tabular}} & \multirow{2}{*}{$\begin{array}{c}\text { After } 6 \mathbf{m} \\
8(40 \%) \\
\end{array}$} & \multirow{2}{*}{$\begin{array}{c}\begin{array}{c}\text { Chi- } \\
\text { square } \\
\text { test }\end{array} \\
27.336\end{array}$} & \multirow{2}{*}{$\begin{array}{l}\mathbf{X}^{2} \mathbf{p} \\
0.000\end{array}$} \\
\hline \multirow{8}{*}{$\begin{array}{l}\text { Endoscopic } \\
\text { criteria }\end{array}$} & \multirow{4}{*}{$\begin{array}{l}\text { Esophageal } \\
\text { Varices }\end{array}$} & \multicolumn{2}{|l|}{ None } & $\begin{array}{c}\text { Number } \\
0\end{array}$ & $\begin{array}{c}\% \\
0.0 \%\end{array}$ & & & & \\
\hline & & \multirow{3}{*}{ Present } & small & 2 & $10.0 \%$ & $7(35 \%)$ & $10(50 \%)$ & & \\
\hline & & & Medium & 15 & $75.0 \%$ & $9(45 \%)$ & $2(10 \%)$ & & \\
\hline & & & Large & 3 & $15.0 \%$ & $1(5 \%)$ & $0(0 \%)$ & & \\
\hline & \multicolumn{3}{|c|}{ Fundal varices } & 1 & $5.0 \%$ & $1(5 \%)$ & $1(5 \%)$ & 0.000 & 1.000 \\
\hline & \multirow{3}{*}{ PHG } & \multicolumn{2}{|c|}{ Non } & 0 & $0.0 \%$ & $7(35 \%)$ & $13(65 \%)$ & \multirow[t]{3}{*}{26.336} & \multirow[t]{3}{*}{0.000} \\
\hline & & \multicolumn{2}{|c|}{$\overline{M i l d}$} & 7 & $35.0 \%$ & $9(45 \%)$ & $6(30 \%)$ & & \\
\hline & & \multicolumn{2}{|c|}{ Severe } & 13 & $65.0 \%$ & $4(20 \%)$ & $1(5 \%)$ & & \\
\hline
\end{tabular}

\section{DISCUSSION}

Budd-Chiari syndrome (BCS) is a rare and lifethreatening vascular disorder, consisting of hepatic venous outflow obstruction at any level between the small hepatic veins and the right atrium [1].

For more than two decades, TIPS has been successfully used for the management of complications of portal hypertension. Given the rarity of $\mathrm{BCS}$, there are no randomized controlled trials (RCT) precisely identifying the timing and candidates for TIPS in BCS. The two common indications established for patients with cirrhosis with portal hypertension (refractory ascites and recurrent variceal bleeding) are common indications for TIPS in patients with BCS [16].
The main aim of our study was to evaluate the effect of TIPS as a decompression procedure -on the portal hypertension as reflected by Grades of esophageal \& gastric varices and Grades of portal hypertensive gastropathy (PHG) in Egyptian patients with BCS.

To our knowledge, this is the first study reported in the literature to evaluate the effect of TIPS on the portal hypertension that is caused by BCS depending on the endoscopic findings after TIPS.

In the current work, large varices were found in 2 patients (10\%), 15 had medium sized varices $(75 \%)$ and 3 had small sized varices $(15 \%)$. This is different from previous results reported by Dabbous and collagues in Egyptian patients with $\mathrm{BCS}$, who found that large size varices were found in $40 \%$ of the patients at the time of diagnosis, $40 \%$ for medium sized and $20 \%$ for small sized varices [6]. In the present study, we 
have recruited 20 patients who had all hepatic veins occlusions, patent PV \& IVC that enabled us to perform the TIPS procedure. Fortunately, enough, the procedure was feasible and completed successfully in all patients (100\%) despite the technical difficulties faced, like absence of a hepatic venous stump, thus requiring a direct puncture from the intrahepatic portion of the IVC. Overall, these data show that, in experienced hands, TIPS can be successfully applied to most patients with severe BCS.

Keeping in mind, that any intervention has its complication, TIPS has its complications which are reported worldwide. Post TIPS transient right upper quadrant abdominal pain and tenderness (lasting 2-3 days after the procedure) was encountered in all studied patients. Post procedure TIPS bleeding (Intraperitoneal) was encountered in 2 patients (10\%); managed successfully by temporal stoppage of anticoagulation and blood transfusion. These complications could be attributed to multiple hepatic puncture during maneuver. Insertion of Gelfoam strips can prevent bleeding from the puncture track after transhepatic intervention [11].

Percutaneous transhepatic technique is more simple than transjugular or transfemoral technique but with a potential risk of bleeding [12]. Post TIPS infection occurred in 2 patients (10\%). Sepsis was diagnosed with fever, elevated leucocytic count with neutrophilia and positive blood culture (Klebsiella).

Post TIPS hepatic encephalopathy occurred in none of our studied patients. Hepatic encephalopathy is well- known complication after TIPS and according to Rosado and Kamath's update on TIPS, new or worsened encephalopathy has been reported in large prospective studies in the range of $13-44 \%$ [13]. Post procedure, immediate stent dysfunction and Stent migration also were not reported in any patient in the present study. Later postoperative complications were shunt occlusion developed 3 months after the intervention in one patient $(5 \%)$ and in another patient at the 6th month (5\%), so the total number of patients, who needed revision with angioplasty and application of another stent were 2 patients $(10 \%)$.

The results of our patients were much better than what have been reported earlier by Valla, who stated that stent occlusion needing reintervention occurs in about $70 \%$ of cases by 6 months [14].
Again, our results were better than what was reported by Senzolo and colleagues, who mentioned that $36-72 \%$ of patients needed sessions of dilatation after TIPS [15].

For more than two decades, TIPS has been successfully used for the prophylaxis and treatment of portal hypertension complications [16]. Successful TIPS insertion effectively lowers the portosystemic pressure gradient, resolves ascites and improves esophageal varices, PHG, liver and renal function [17]. TIPS was successful in reducing portal pressure and preventing variceal bleeding and ascites in $99 \%$ of patients [18].

In patients with acute variceal bleeding and high risk of early rebleeding, recent studies showed improved survival recommending early TIPS implantation. With respect to the prevention of rebleeding (secondary prophylaxis), in recent meta-analysis, which included twenty-four studies (1120 subjects treated with TIPS and 1065 subjects treated with ET), Zhang and colleagues has concluded that TIPS with covered stents might be considered the preferred choice of therapy in patients with severe liver disease for secondary prophylaxis [19].

As demonstrated in 2 relevant meta-analyses, TIPS improve survival in patients with refractory ascites, justifying its earlier application. It clearly reverses the circulatory dysfunction, which leads to normalization of the renal function. With a 10year survival rate of $80 \%$, TIPS is the preferred treatment in patients with Budd-Chiari syndrome who do not respond sufficiently to medical treatment [20]. In the current study, we clarify the impact of TIPS on the clinical, laboratory, radiological and endoscopic findings of the studied patients who had primary BCS. We compared between Laboratory investigations before intervention, during and at the end of follow up. We reported a significant decreased ALT level, serum Bilirubin, insignificant decreased AST level, significant increased Serum albumin after TIPS. Also, we detected improvement of the Child- Pugh score after TIPS. This improvement of synthetic function of the liver might be related to relieve of hepatic congestion. The mean size of the liver and spleen $(\mathrm{cm})$ at the end of follow up was decreased significantly when compared with that before the intervention.

The mean portal vein diameter was decreased significantly and the mean portal vein velocity

Abdelmoaty et al., Afro-Egypt J Infect Endem Dis 2021;11(1):18-26

https://aeji.journals.ekb.eg/

http://mis.zu.edu.eg/ajied/home.aspx 
was increased significantly at the end of follow up. The frequency of hepatic-petal portal vein flow direction significantly increased at the end of follow up.

TIPS was equally effective at controlling bleeding from gastric as well as esophageal varices. Controlled trials comparing surgical shunts or glue in the treatment of these patients would help to better define the role of TIPS in the management of patients with bleeding from gastric varices [2]. In our study, endoscopic findings of esophageal varices among the studied patients with EV, (Before TIPS: total $n=3 / 20$ had large EV, 15 had medium sized EV and 2 had small EV) showed a highly statistically significant improvement of EV after TIPS. In two of three patients with large EV within onemonth post-TIPS ( 2 reduced to medium sized) and in all 3 patients within 6 months post-TIPS (2 large reduced to medium sized and one to small EV). 15 patients with medium sized EV markedly improved to 7 patients reduced to small $\mathrm{EV}$ and one reduced to non $\mathrm{EV}$ in one month and 9 patients to small EV and 6 reduced to non EV after 6 months and all 2 patients with small $\mathrm{EV}$ reduced to non $\mathrm{EV}$ after one and six months. Isolated gastric varix was persisted at one and six months. Regarding endoscopic findings of PHG before and after TIPS in the current study, among the studied patients with PHG $(n=20), 13$ had severe PHG and 7 had mild PHG. After TIPS, there was a highly statistically significant improvement of PHG in most cases. 9 out of the thirteen patients with severe PHG within one-month post-TIPS (reduced to mild disease) and in 12 of 13 within 6 months postTIPS ( 6 reduced to mild disease and 6 reduced to none). All 7 patients with mild PHG markedly improved to non PHG in one and six months.

\section{CONCLUSION}

TIPS is a safe and technically feasible treatment option for patients with BCS. Moreover, TIPS was successful in improving liver profile, controlling ascites, and improving esophageal varices \& PHG.

\section{Ethical Considerations:}

All patients provided an informed written consent and the study protocol was approved by the Research Ethical Committee of Faculty of Medicine, Ain Shams University according to the ethical guidelines of the 1975 Declaration of Helsinki.

Funding: None.

\section{Conflict of interest: None}

\section{REFERENCES}

1. Valla DC. Primary Budd-Chiari syndrome. Journal of Hepatology 2009; 50: 195-203.

2. De Franchis R. Evolving consensus in portal hypertension. Report of the Baveno IV consensus workshop on methodology of diagnosis and therapy in portal hypertension. J Hepatol 2005; 43: $167-176$.

3. Valla DC. Hepatic vein thrombosis (Budd-Chiari syndrome). Semin Liver Disease 2002; 22: 5-14.

4. Kamath P, Wiesner R, Malinchoc M, Kremers W, Therneau T, Kosberg C, et al. A model to predict survival in patients with end-stage liver disease. Hepatology 2001; 33: 464-470.

5. Slakey DP, Klein AS, Venbrux AC, Cameron JL. Budd Chiari Syndrome. Current management options., Annals of Surgery 2001; 233 (4): 512517.

6. [6] Dabbous H, Sakr M, Abdelhakam S, Youssef S, Gharib M, Shaker M, et al. Feasibility of Anticoagulation in Patients of Budd-Chiari Syndrome with Gastroesophageal Varices and Portal Hypertension. Journal of GHR 2013; 21 2(5): 581-584.

7. Qi X, He C, Yin Z, Wangl Z. PVT-TIPS Study Group: Transjugular intrahepatic portosystemic shunt for the prevention of variceal rebleeding in cirrhotic patients with portal vein thrombosis: study protocol for a randomized controlled trial. BMJ Open 2013; 3(7): e003370.

8. Bosch J, Abraldes J and Groszmann R. Current management of portal hypertension. J Hepatol 2003; 38: Suppl 1:S54-S68.

9. Garcia-Tsao G, Sanyal AJ, Grace ND, Carey W. Prevention and management of gastroesophageal varices and variceal hemorrhage in cirrhosis. i2007; 46: 922-938.

10. De Franchis R. Updating consensus in portal hypertension: report of the Baveno III Consensus Workshop on definitions, methodology and therapeutic strategies in portal hypertension 2000; 33(5):846-52.

11. Eapen CE, Velissaris D, Heydtmann M, B Gunson, S Olliff, E Elias. Favorable medium term outcome following hepatic vein recanalisation and/or transjugular intrahepatic portosystemic shunt for Budd Chiari syndrome. Gut 2006; 55 (6): 878-884. 
12. Beckett D, Olliff S. Interventional radiology in the management of Budd Chiari syndrome. Cardiovasc Intervent Radiol 2008; 31:839-47.

13. Rosado B ' Kamath PS. Transjugular intrahepatic portosystemic shunts: an update. Liver Transpl 2003; 9 (3): 207-17.

14. Valla D. The diagnosis and management of the Budd-Chiari syndrome: consensus and controversies. Hepatology 2003; 38:793-803.

15. Senzolo M, Cholongitas EC, Patch D and Burroughs AK. Update on the Classification, Assessment of Prognosis and Therapy of BuddChiari Syndrome. Nat Clin Pract Gastroenterol Hepatol 2005; 2(4):182-190.

16. Boyer D , Haskal J. The Role of Transjugular Intrahepatic Portosystemic Shunt (TIPS) in the Management of Portal Hypertension: Update 2009. Hepatology 2010; 51(1): 306-316.
17. Qi X, Ren W, Wang Y, Guo X, Fan D. Survival and prognostic indicators of Budd-Chiari syndrome: a systematic review of 79 studies. Expert Rev Gastroenterol Hepatol 2015; 9: 865-875.

18. Tripathy D, MacNicolas R, Kothari C, Sunderraj L, Al-Hilou H, Rangarajan B, et al. Good clinical outcomes following transjugular intrahepatic portosystemic stent-shunts in Budd-Chiari syndrome. Aliment Pharmacol Ther 2014; 39: 864-872.

19. Zhang $\mathrm{H}$, Zhang $\mathrm{H}, \mathrm{Li} \mathrm{H}$. TIPS versus endoscopic therapy for variceal rebleeding in cirrhosis: A meta-analysis update. J Huazhong Univ Sci Technolog Med Sci 2017; (4):475-485.

20. Rossle M. TIPS: 25 years later. Journal of Hepatology 2013; 59 (5): 1081-1093.

21. Tripathi D, Therapondos G, Jackson E, Redhead DN ,Hayes PC. The role of the transjugular intrahepatic portosystemic stent shunt (TIPSS) in the management of bleeding gastric varices: clinical and haemodynamic correlations. Gut 2002; 51:270-274. 